\title{
Stakeholders Analysis: Um novo campo de pesquisa no turismo
}

\author{
Stakeholders Analysis: A new field of research in tourism
}

\section{Stakeholders Analysis: Un nuevo campo de investigación en turismo}

\author{
Saulo Fabiano Amâncio Vieira ${ }^{1}$ \\ Benny Kramer Costa ${ }^{2}$ \\ Renato Fabiano Cintra ${ }^{3}$
}

\begin{abstract}
Resumo: O presente artigo buscou realizar um levantamento bibliográfico acerca dos trabalhos que versam sobre stakeholders analysis, bem como identificar possíveis temas de pesquisa na área do turismo. A pesquisa foi realizada em janeiro de 2010, utilizando-se da base de referências web of science, organizada de acordo com seu fator de impacto da publicação, focando no tema stakeholders analysis, onde foram encontrados 1.296 artigos, a partir desses foram selecionados os 200 mais relevantes (por fator de impacto), a qual foi feita uma análise prévia de seu conteúdo e separou aqueles que efetivamente abordavam aspectos teóricos da stakeholders analysis, perfazendo um rol de 51 artigos. Em relação a sua temática central, levantou-se: Meio Ambiente (13), Redes de Relacionamento (8), Tomada de Decisão (9), Políticas Públicas (6), Gestão de Projetos (4) e Revisão de literatura (11). Predomina nestes artigos a abordagem qualitativa com 42 (82\%). Verifica-se o crescente interesse sobre a utilização da temática de stakeholders analysis, a partir de 2005 a periodicidade de publicação destes artigos foi intensificada (39 artigos), demonstrando o potencial e maturidade da teoria. Também observou que existem diferentes abordagens teóricas de stakeholders analysis que podem ser exploradas em pesquisas no turismo, sendo encontrados 5 artigos abordando a temática.
\end{abstract}

Palavras-chave: Stakeholders analysis; Stakeholders no Turismo; Teoria de Stakeholders; Turismo; Estratégia.

\begin{abstract}
This article attempts to make a bibliography on works about the stakeholder analysis and identify possible research topics in tourism. The survey was conducted in January 2010, using the base of science web of references, organized according to their impact factor of publication, focusing on the theme stakeholder analysis, which found 1,296 articles from 200 of these were selected most relevant (by impact factor), which was made a preliminary analysis of its contents and addressed effectively separated those theoretical aspects of stakeholder analysis, making a list of 51 articles. In relation to its central theme, rose: Environment (13) Social Networking (8), Decision Making, (9) Public Policy (6), Project Management (4) and Literature Review (11). Predominates in these articles the qualitative approach with 42 (82\%). There is growing interest on the use of thematic analysis of stakeholders, from 2005 the frequency of publication of these articles have been stepped up (39 articles), demonstrating the potential and maturity of the theory.

\footnotetext{
${ }^{1}$ Doutor em Administração pela Uninove/SP e Professor do Programa de Pós-Graduação em Administração da Universidade Estadual de Londrina (PPGA/UEL).E-mail: saulo@uel.br

2 Pós-doutor e Doutor em Administração pela FEA/USP e Professor do Programa de Mestrado e Doutorado da Universidade Nove de Julho (PMDA-UNINOVE) e da ECA-USP.E-mail: bennycosta@yahoo.com.br

3 Mestrando do Programa de Pós-Graduação em Administração da Universidade Estadual de Londrina (PPGAUEL). Email: renatocintra@hotmail.com
} 
He also noted that there are different theoretical approaches to stakeholder analysis that can be exploited in research on tourism, and found five articles addressing the topic.

Keywords: Stakeholders Analysis; Stakeholders in Tourism; Theory of Stakeholders; Tourism; Strategy.

Resumen: En este artículo se intenta hacer una bibliografía de trabajos sobre stakeholders analysis $e$ identificar posibles temas de investigación en turismo. La investigación fue realizada en enero de 2010, utilizando la base web of science, se centra en el stakeholders analysis del tema, que se encuentran 1.296 artículos de 200 de éstos fueron seleccionados más relevantes (factor de impacto), que se hizo un análisis preliminar de su contenido y abordarse con eficacia separaba a los aspectos teóricos del stakeholders analysis, haciendo una lista de 51 artículos. En relación a su tema central, se levantó: el Medio Ambiente (13) Redes Sociales (8), la Toma de Decisiones, (9) Políticas Públicas (6), Gestión de Proyectos (4) y la Revisión de la Literatura (11). Predomina en estos artículos de la aproximación cualitativa con 42. Existe un interés creciente en el uso de análisis temático de stakeholders analysis, a partir de 2005 la frecuencia de publicación de estos artículos se han intensificado (39), lo que demuestra el potencial y la madurez de la teoría. También señaló que hay diferentes enfoques teóricos del stakeholders analysis que pueden ser explotadas en la investigación sobre el turismo, y encontraron cinco artículos que abordan el tema.

Palabras clave: Stakeholders Analysis; Stakeholders en el Turismo; Teoria de los Stakeholders; Turismo; Estrategia.

\section{INTRODUÇÃO}

Desde 1984, observa-se um crescente interesse sobre a temática stakeholders, ano este que Richard E. Freeman publica Strategic Management: a Stakeholder Approach, que passou abordar na literatura gerencial a gestão dos stakeholders como um fator crítico de sucesso das organizações. Freeman (1984) relata que a suposição principal da teoria dos stakeholders é aquela onde a eficácia da organização é medida por sua habilidade de satisfazer não somente os acionistas, mas igualmente aqueles agentes que têm um vínculo com a organização.

No que se refere às definições do termo stakeholders, 55 delas foram levantadas em 75 publicações, entre os anos de 1963 e 2003, por Friedman e Miles (2006), sendo que todas tomam a organização como o centro das discussões. A definição clássica de stakeholder é "qualquer grupo ou pessoa cujos interesses podem afetar ou ser afetados pelas realizações dos objetivos de uma organização" (FREEMAN, 1984, p. 46).

A partir de então diversos periódicos acadêmicos de renome internacionais como Business Ethics Quartely, Critical Perspectives in Accounting, Academy of Management Review e Academy of Management Journal dentre outros, têm se dedicado a elaboração e discussão do assunto, assim como tem lançado diversos questionamentos na área (FRIEDMAN e MILES, 2006). Estes aspectos, em seu conjunto, têm contribuído para a evolução dos estudos relacionados ao tema, assim como permite induzir a realização de novos trabalhos em organizações e setores econômicos até então pouco explorados ou sem ter sido alvo de qualquer estudo em relação à atuação de seus stakeholders.

Tendo como ponto de partida as considerações expostas, o presente artigo tem como objetivo realizar um levantamento bibliográfico acerca dos trabalhos que versam sobre 
stakeholders analysis, bem como identificar possíveis temas de pesquisa na área do turismo. Para tanto, o artigo está assim estruturado: após a introdução, são apresentados os procedimentos metodológicos e em seguida, a revisão de literatura realizada. Por fim, são apresentadas as considerações.

\section{PROCEDIMENTOS METODOLÓGICOS}

O estágio inicial da presente revisão desenvolveu uma fundamentação preliminar com os conceitos chave da teoria dos stakeholders utilizando-se de autores clássicos e de livros seminais acerca da teoria. Posteriormente foi dado prosseguimento a uma revisão geral do tema conforme procedimentos descritos a seguir, considerando ser esta uma pesquisa descritiva - exploratória. Caracteriza também com uma pesquisa documental, pois buscou analisar a produção científica a cerca do tema proposto.

Inicialmente foi realizada uma pesquisa no ISI - Web Of Science (WOS). O ISI é um índice de citações na web, multidisciplinar que indexa mais de 9200 revistas ou publicações periódicas. Esta base indexa todos os itens de relevância e é atualizada semanalmente.

A pesquisa foi realizada durante o mês de julho de 2009 onde se encontrou 4.533 artigos a partir da expressão stakeholder. Posteriormente a busca foi refinada incluindo os termos stakeholder theory, onde encontrou-se 732 artigos; stakeholder analysis encontrando-se 1.209 artigos e stakeholder management com 2.105 artigos publicados.

Em janeiro de 2010 realizou-se nova busca, organizada de acordo com seu fator de impacto da publicação, só que agora focando no tema stakeholder analysis, já que esta é a temática central da presente pesquisa, onde foram encontrados 1.296 artigos, a partir desses foram selecionados os 200 mais relevantes (por fator de impacto) que possuíam como foco central do trabalho stakeholders analysis. Nesses 200 artigos foi realizada uma análise prévia de seu conteúdo do qual se separou aqueles que efetivamente abordavam aspectos teóricos da stakeholders analysis, perfazendo um rol de 51 artigos, sendo que os mesmos são descritos nas próximas seções. A partir dos procedimentos descritos acima, procedeu ao desenvolvimento da presente revisão.

\section{STAKEHOLDERS ANALYSIS: REVISANDO A LITERATURA}

Sobre a pesquisa geral da Teoria dos Stakeholders mais especificamente Stakeholder Analysis, conforme descritos na metodologia para a realização desta revisão foram selecionados os 51 artigos que possuíam como temática e foco central Stakeholder Analysis. Tais categorias podem ser observadas nos trabalhos de Freeman (1984), Mitroff (1983), Reed, Graves e Dandy (2009), Mitchell, Agle e Wood (1997). Inicialmente os trabalhos foram categorizados conforme foco central da Análise de Stakeholders conforme destacado na Figura 1: 


\begin{tabular}{|c|l|}
\hline Análise de Stakeholders : Foco & \multicolumn{1}{|c|}{ Autores } \\
\hline Identificação & $\begin{array}{l}\text { Hermans (2008), Currie, Seaton e Wesley (2009), Billgren e Holmen (2008), Jepsen e Eskerod (2009), } \\
\text { Wu (2007), Fassin (2008), Reed, Graves e Dandy (2009), Pomero e Douvere (2008) e Fassin (2009) }\end{array}$ \\
\hline Interesses e expectativas & $\begin{array}{l}\text { Jepsen e Eskerod (2009), Pomero e Douvere (2008), Kooskora (2008), Cameron, Craweley e Loureiro } \\
\text { (2008), Friedman e Mason (2004) e Robson e Robson (1996) }\end{array}$ \\
\hline Influência & $\begin{array}{l}\text { Prell, Hubacek e Reed (2009), Ferrary (2009), Yau, Chow e Sin (2007), Cheng, Millar e Choi (2006), } \\
\text { Hendry (2005), Reed, Graves e Dandy (2009) e Hermans e Thissen (2009) }\end{array}$ \\
\hline \multirow{2}{*}{ Tomada de decisão } & $\begin{array}{l}\text { Chase, Siemer e Decker (2002), Linkov, Satterstrom e Kiker (2006), Simmons, Tles e Yolles (2005), } \\
\text { Schwarzkopf (2006), Beierle (2002), Wu (2007), Brown, Adger e Tompkins (2001), Reed (2008), } \\
\text { Tompkins, Few e Brown (2008), Friedman e Mason (2004) e Magness (2008) }\end{array}$ \\
\hline Relacionamentos & $\begin{array}{l}\text { Weible (2007), Zakhem (2008), Ferrary (2009), Chung, Chen e Reid (2009), Williams e Lewis (2008), } \\
\text { Kooskora (2008), Reed, Graves e Dandy (2009), Friedman e Miles (2002), Robson e Robson (1996), } \\
\text { Simmons e Lovegrove (2005) e Roloff (2008) }\end{array}$ \\
\hline Poder e legitimidade & \begin{tabular}{l} 
Bilgren e Holmen (2008), Friedman e Mason (2004) e Hsieh (2009) \\
\hline Outros
\end{tabular} \\
$\begin{array}{l}\text { De Lopez (2001), Pan (2005), Lim, Ahn e Lee (2005), Wolfe e Putler (2002), Co e Barro (2009), } \\
\text { Varvasovszky e Brugha (2000), Vandekerckhove e Dentchev (2005), Lamberg, Pajunen e Parvinen } \\
\text { (2008), Web e Raffaelli (2008), Medilanski, Chuan e Mosler (2007), Michalski e Cousins (2001), } \\
\text { Boaventura et al. (2009), Yang, Shen e Ho (2009), Wong (2005) e Vidgen (1997) }\end{array}$ \\
\hline
\end{tabular}

Figura 1. Foco central na análise de stakeholders Fonte: Elaboração própria.

Ao categorizar os artigos em relação ao foco da análise de stakeholders, foram listados os seguintes temas: identificação, interesses e expectativas, influência, tomada de decisão, relacionamentos, poder e legitimidade, e outros aspectos.

Levantou-se que nove trabalhos focaram o processo de identificação dos stakeholders, abordando a identificação e interação, identificação e categorização, identificação e compreensão, a sistematização do processo de identificação e a importância da identificação.

Já o processo de levantamento dos interesses e expectativas obteve seis trabalhos na análise dos stakeholders. Busca saber como revelar as expectativas e interesses (não somente de forma conceitual), balancear as atividades organizacionais e os interesses sociais, identificação do valor percebido dos stakeholders e nos interesses que realmente são relevantes.

Em relação a categoria influência dos stakeholders como foco central, busca compreender como se dá a influência entre os stakeholders e os tipos de influência exercidos, que é outro aspecto levantado nas pesquisas bem como esta relacionada com o processo de tomada de decisão a ser explanado na sequência.

No que tange a tomada de decisão os trabalhos referiam-se desde a qualidade das decisões, incorporação da perspectiva do stakeholder, envolvimento, transparência e diversidade de valores, aspectos estes identificados nos trabalhos citados.

Os trabalhos sobre relacionamentos entre stakeholders destacaram-se o nível de relacionamento entre os próprios stakeholders, bem como com o ambiente, a extensão de tais relações e sua evolução ao longo do tempo, a complexidade do sistema de relacionamentos e as relações de dependência de recursos.

Tem-se ainda que é abordado na análise de stakeholders as questões de legitimidade e poder dos stakeholders, abordando a desigualdade do mesmo entre partes interessadas e mostrando, através de diagramas, a importância relativa do poder de tomada de decisão. Foram 
identificados ainda outros fatores nos trabalhos analisados a saber: participação / envolvimento dos stakeholders nos processos da organização, percepção dos stakeholders, fatores críticos de sucesso associados com a gerência de stakeholders, subordinação da função-objetivo dos stakeholders com a dos shareholders, características e definição de stakeholder.

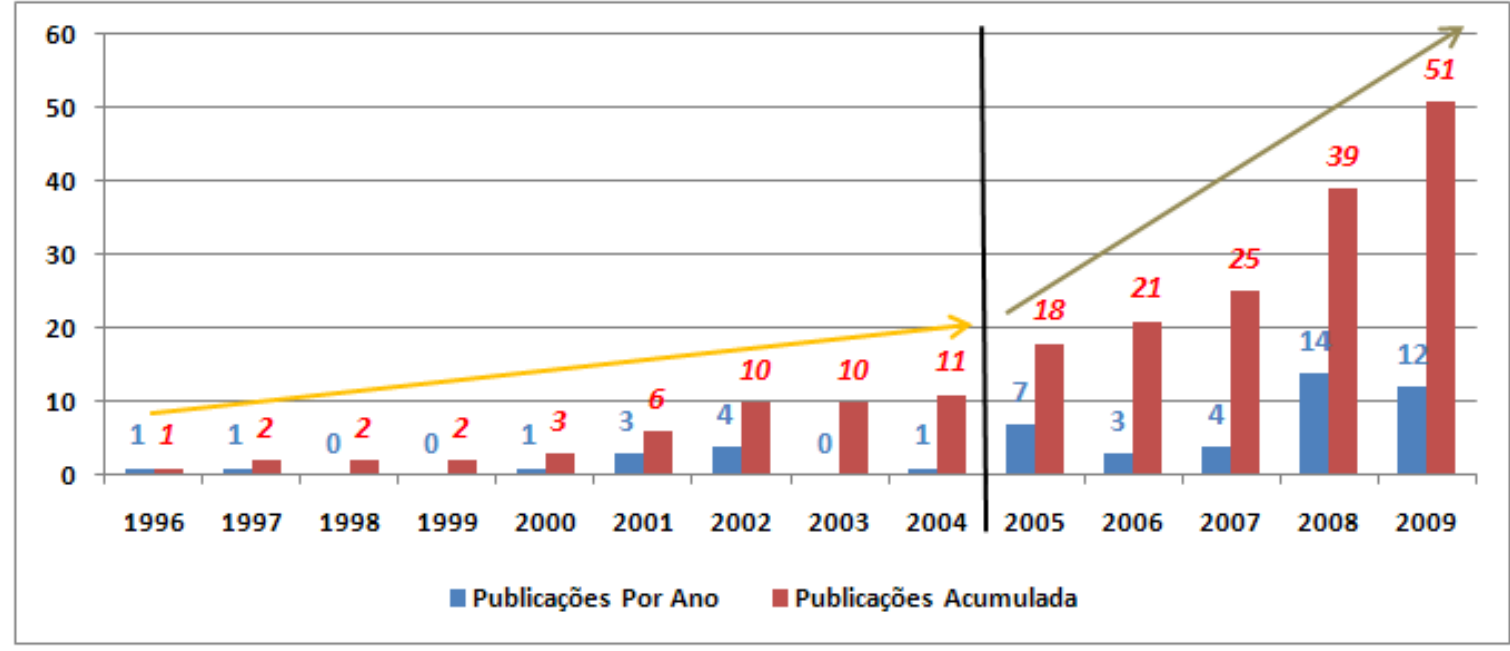

Figura 2. Abordagens metodológicas nas pesquisas do turismo Fonte: Elaboração própria.

No que tange aos 51 artigos analisados, eles variam quanto ao período em que foi publicado de 1996 até 2009. O ano que teve a maior quantidade publicada corresponde ao ano de 2008 com 14 artigos. Pode destacar que conforme Figura 2, as publicações existem dois períodos a considerar: o primeiro engloba 1996 até o ano de 2004 (11 trabalhos) e o segundo a partir do ano de 2005 até 2009 (40 trabalhos), sendo que neste segundo período a periodicidade de publicação destes artigos foi intensificada (40 ou 78,4\%). Vale frisar que 1998, 1999 e 2003 não houve nenhuma publicação no que diz respeito à amostra estudada.

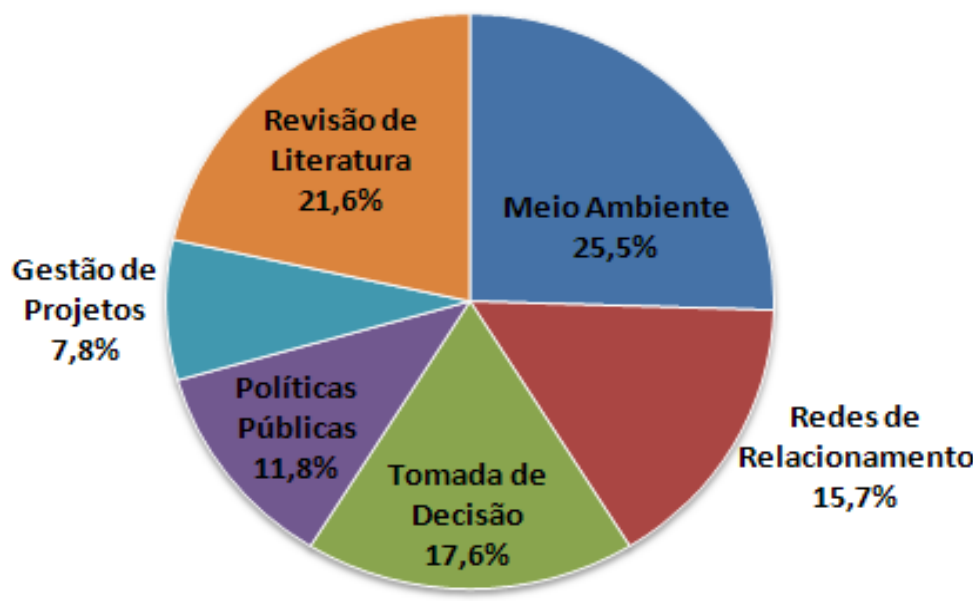

Figura 3. Temática central Fonte: Elaboração própria. 
Em relação a sua temática central (Figura 3), levantou-se: Meio Ambiente (13 ou 25,5\% do total), Redes de Relacionamento (8 artigos ou 15,7\%), Tomada de Decisão (9 artigos ou 17,6\%), Políticas Públicas (6 artigos ou 11,8\%), Gestão de Projetos (4 artigos ou 7,8\%) e Revisão de Literatura (11 artigos ou 21,6\%).

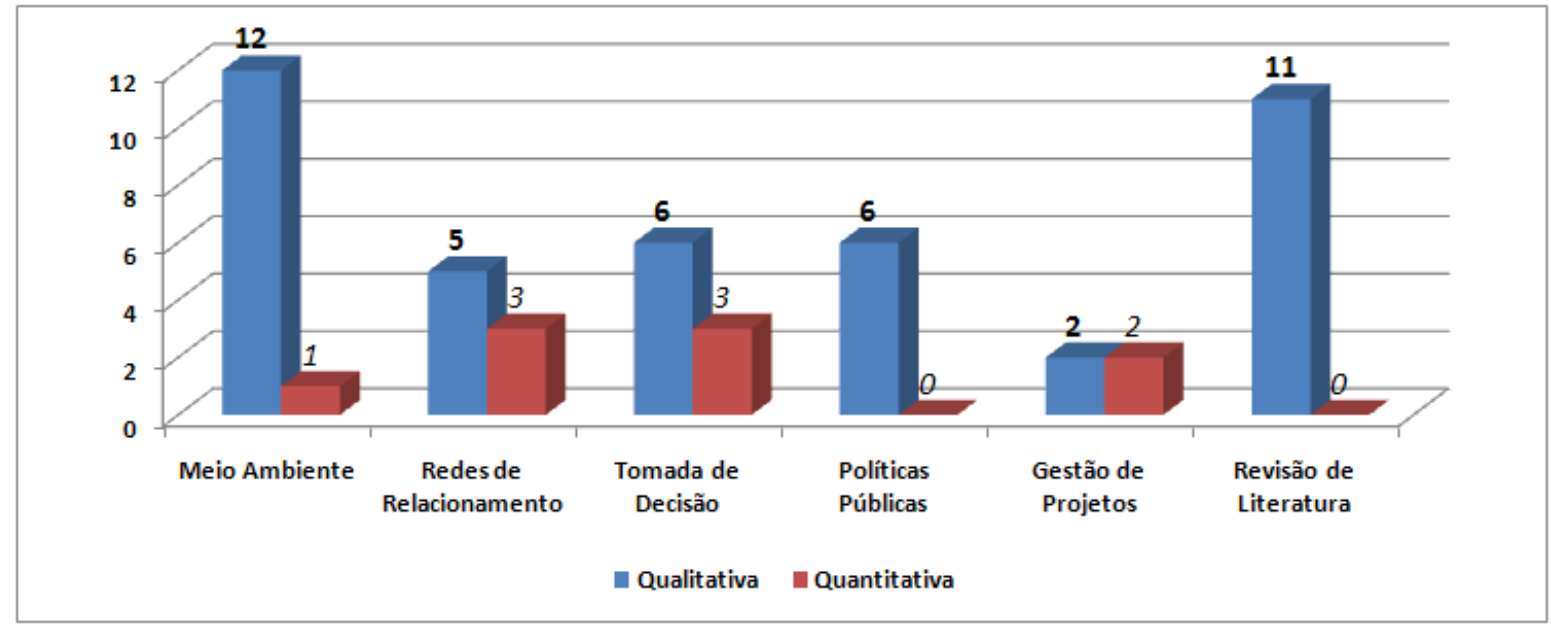

Figura 4. Temática central e suas respectivas abordagens metodológicas Fonte: Elaboração própria.

Sobre o tema Meio Ambiente (Figura 4) foram identificados (13 artigos), dos quais doze possuem abordagem metodológica qualitativa (92,3\%), sendo apenas um quantitativo $(7,7 \%)$. Foram temas destes trabalhos: gestão dos recursos naturais, meio ambiente e política, desenvolvimento sustentável, gerência de agências estatais, gestão ambiental e valores dos stakeholders.

Na temática Rede de Relacionamento (Figura 4), oito artigos foram produzidos dos quais cinco qualitativos (62,5\%) e três quantitativos (37,5\%). Abordaram interações dos empreendedores, processo de fusão, dinâmica das interações, relacionamentos, percepção dos líderes empresariais, necessidades de relacionamento e tipos e a importância dos relacionamentos.

Em relação à Tomada de Decisão (Figura 4), tem-se que nove artigos foram identificados dos quais seis foram qualitativos $(66,7 \%)$ e três quantitativos (33,3\%). Verificaram-se os seguintes assuntos: decisões ambientais, incorporação da perspectiva dos stakeholders na tomada de decisão, formas de participação dos stakeholders na tomada de decisão, tomada de decisão no setor público, tomada de decisão estratégica e orientação estratégica para stakeholders.

No que tange às Políticas Públicas (Figura 4), tem-se seis artigos, todos utilizando abordagens qualitativas (100\%). Dentre os temas destes artigos verificou-se: análise política dos stakeholders, motivações dos subsistemas políticos composto pelos stakeholders, a importância dos stakeholders no setor público, sustentação das decisões políticas e interesses dos stakeholders.

Sobre o tema Gestão de Projetos quatro artigos foram identificados (Figura 4), sendo dois 
qualitativos (50\%) e dois quantitativos (50\%). Foram abordadas as possíveis limitações da análise de stakeholders em projetos, problemas no desenvolvimento de software, fatores críticos de sucesso em projetos de construção civil e melhoria na qualidade de software.

Identificaram-se ainda onze artigos sobre Revisão de Literatura (Figura 4), os quais versaram sobre modelos de análise, tipos de estratégias, desenvolvimento de modelos, representações gráficas, debates teóricos sobre definições, recursos humanos e aproximações participativas.

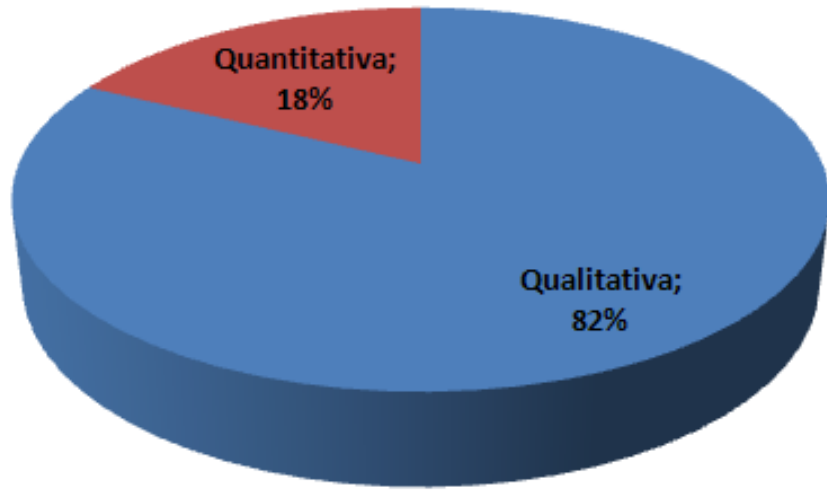

Figura 5. Abordagens metodológicas Fonte: Elaboração própria.

Houve uma preponderância da abordagem metodológica qualitativa nestes trabalhos (42 artigos ou $82 \%$ do total analisado), sendo utilizadas diferentes estratégias, a saber: estudo de caso único e duplo, grounded theory, análise longitudinal, grupo de foco. Já na abordagem quantitativa (9 artigos ou 18\% do total analisado) observou-se a utilização de surveys, análise multicritério e estatísticas descritivas, conforme Figura 5.

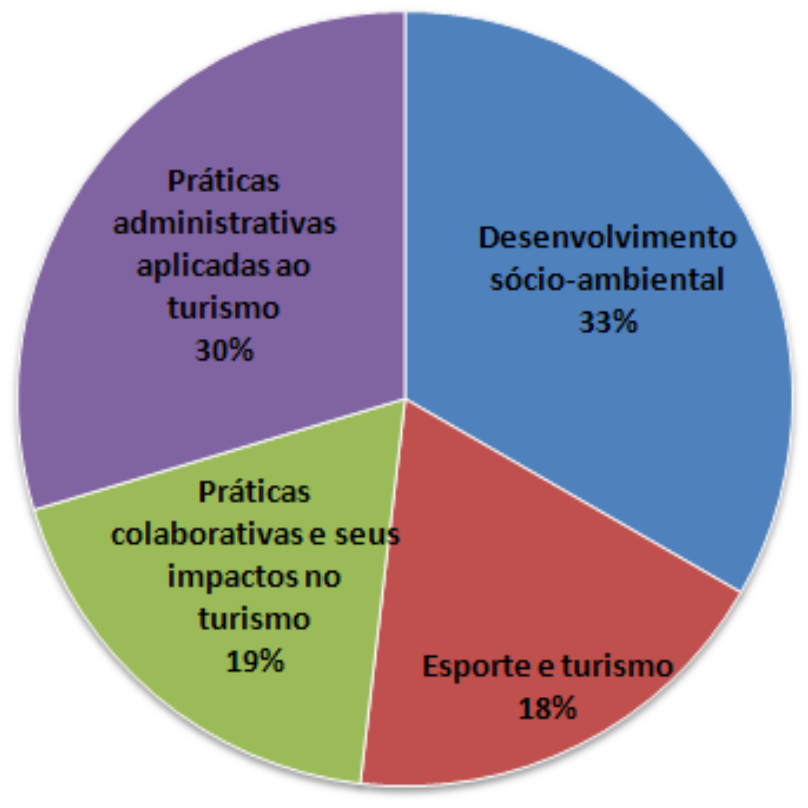

Figura 6. Temática central de pesquisa no turismo Fonte: Elaboração própria. 
Ao comparar o presente levantamento com a pesquisa realizada por Vieira et al. (2010) onde os autores realizaram um levantamento bibliográfico acerca dos trabalhos que versam sobre a teoria dos stakeholders aplicados em organizações turísticas, onde procedeu-se uma pesquisa bibliográfica a partir da expressão stakeholder, posteriormente a busca foi refinada incluindo os termos stakeholder theory, stakeholder analysis e stakeholder management, e buscou-se ainda especificamente o tema que tem relação direta com a área do turismo, onde encontrou-se Hospitality, Leisure, Sport \& Tourism (27 artigos) em nove diferentes journals.

Os artigos foram categorizados conforme sua temática central (Figura 6), a saber: desenvolvimento sócio-ambiental (9 artigos), esporte e turismo (5 artigos), práticas colaborativas e seus impactos no turismo ( 5 artigos) e práticas administrativas aplicadas ao turismo (8 artigos).

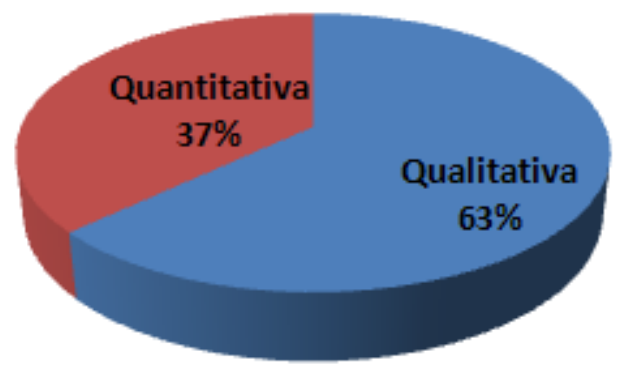

Figura 7. Abordagens metodológicas nas pesquisas do turismo Fonte: Elaboração própria.

Conforme Figura 7, houve uma preponderância da abordagem metodológica qualitativa nestes trabalhos (17 ou 63\%), enquanto que a abordagem metodológica quantitativa apresentou nove (37\%), sendo que a partir de 2005 a periodicidade de publicação destes artigos também foi intensificada (25).

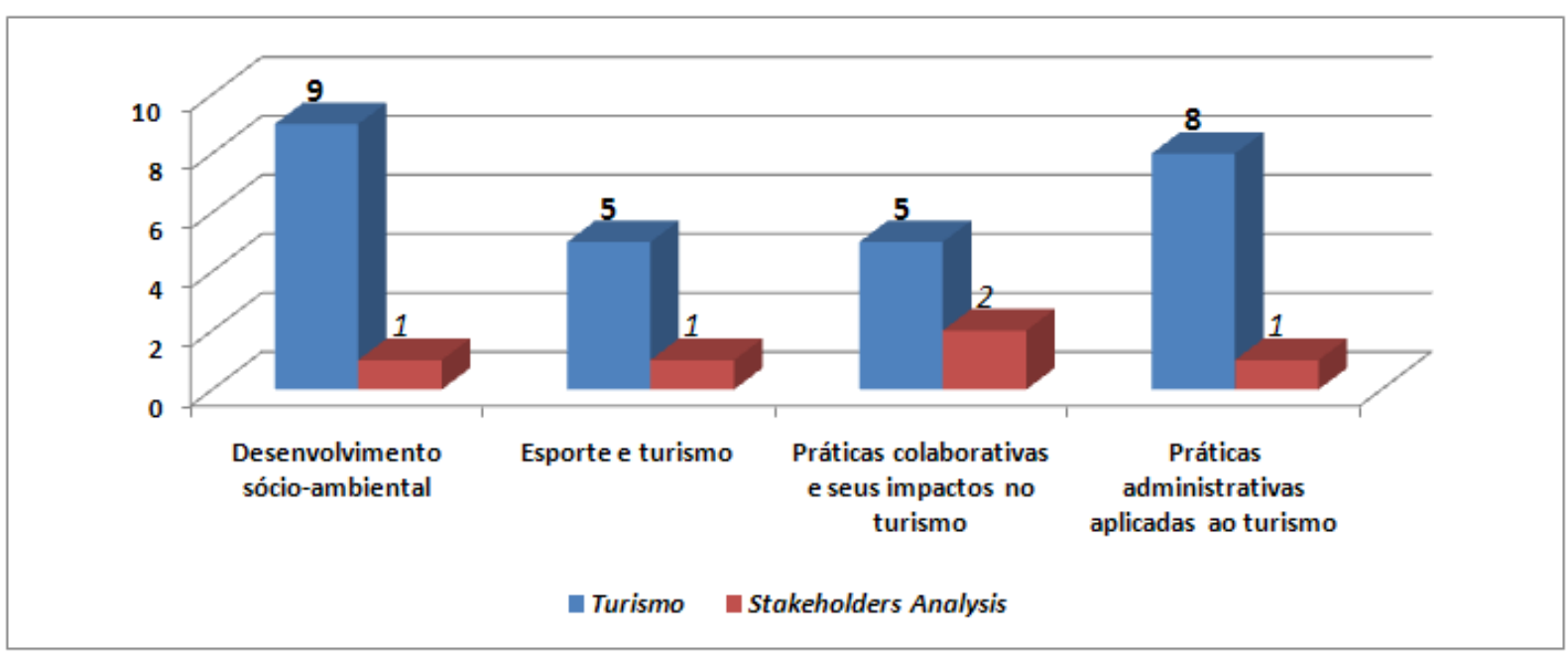

Figura 8. Temática central do turismo e abordagens de stakeholders analysis Fonte: Elaboração própria. 
Assim, conforme Figura 8, observa-se que em relação ao tema desenvolvimento sócioambiental foram produzidos no período analisado 9 (nove) artigos dos quais 1 (um) possuem abordagem focada na análise de stakeholders. O mesmo ocorrendo no tema esporte e turismo com apenas 1 artigo. No que tange ao tema práticas colaborativas e seus impactos no turismo foram produzidos no período analisado 5 (cinco) artigos dos quais 2 (dois) trabalharam a análise de stakeholders. E finalizando, observa-se que em relação ao tema práticas administrativas aplicadas ao turismo foram produzidos no período analisado 8 (oito) artigos dos quais 1 (um) na abordagem da análise, totalizando apenas 5 artigos produzidos no período.

\section{CONSIDERAÇÕES FINAIS}

O presente artigo buscou realizar um levantamento bibliográfico acerca dos trabalhos que versam sobre stakeholders analysis, bem como identificar possíveis temas de pesquisa na área do turismo. Os artigos foram categorizados conforme seu foco bem como sua temática central.

Em relação ao foco da análise de stakeholders ser a identificação verificou-se que nove trabalhos focaram o processo de identificação dos stakeholders, abordando a identificação e interação, identificação e categorização, identificação e compreensão, a sistematização do processo de identificação e a importância da identificação. Tais trabalhos foram aplicados em diferentes contextos sendo que se abordaram ainda os possíveis conflitos gerados por não se levar em consideração os stakeholders nas decisões da organização. Foram desenvolvidos por Hermans (2008), Currie, Seaton e Wesley (2009), Billgren e Holmen (2008), Jepsen e Eskerod (2009), Wu (2007), Fassin (2008), Reed, Graves e Dandy (2009), Pomero e Douvere (2008) e Fassin (2009).

No que tange aos interesses e expectativas dos stakeholders foram levantados seis trabalhos na análise dos stakeholders. Desenvolvido por Jepsen e Eskerod (2009), Pomero e Douvere (2008), Kooskora (2008), Cameron, Craweley e Loureiro (2008), Friedman e Mason (2004) e Robson e Robson (1996), para buscar saber como revelar as expectativas e interesses (não somente de forma conceitual), balancear as atividades organizacionais e os interesses sociais, identificação do valor percebido dos stakeholders e nos interesses que realmente são relevantes.

Em relação a influência dos stakeholders, Prell, Hubacek e Reed (2009), Ferrary (2009), Yau, Chow e Sin (2007), Cheng, Millar e Choi (2006), Hendry (2005), Reed, Graves e Dandy (2009) e Hermans e Thissen (2009) buscaram compreender como se dá a influência entre os stakeholders e os tipos de influência exercidos, que é outro aspecto levantado nas pesquisas bem como está relacionada com o processo de tomada de decisão a ser explanado a seguir.

Sobre o processo de tomada de decisão, ele se referia tanto à qualidade das decisões, incorporação da perspectiva do stakeholder, envolvimento, transparência e à diversidade de valores, aspectos estes identificados nos trabalhos citados. Trabalhos estes desenvolvidos por Chase, Siemer e Decker (2002), Linkov, Satterstrom e Kiker (2006), Simmons, lles e Yolles (2005), Schwarzkopf (2006), Beierle (2002), Wu (2007), Brown, Adger e Tompkins (2001), Reed (2008), 
Tompkins, Few e Brown (2008), Friedman e Mason (2004) e Magness (2008).

Os relacionamentos entre stakeholders estiveram presentes nos trabalhos de Weible (2007), Zakhem (2008), Ferrary (2009), Chung, Chen e Reid (2009), Williams e Lewis (2008), Kooskora (2008), Reed, Graves e Dandy (2009), Friedman e Miles (2002), Robson e Robson (1996), Simmons e Lovegrove (2005) e Roloff (2008). Destaca-se o nível de relacionamento entre os próprios stakeholders, bem como com o ambiente, a extensão de tais relações e sua evolução ao longo do tempo, a complexidade do sistema de relacionamentos e as relações de dependência de recursos.

Ainda são mencionadas na análise de stakeholders as questões de legitimidade e poder dos stakeholders, abordando a desigualdade do mesmo entre partes interessadas e mostrando, através de diagramas, a importância relativa do poder de tomada de decisão. Outros fatores foram identificados nos trabalhos analisados a saber: participação / envolvimento dos stakeholders nos processos da organização, percepção dos stakeholders, fatores críticos de sucesso associados com a gerência de stakaholders, subordinação da função-objetivo dos stakeholders com a dos shareholders, características e definição de stakeholder. Foram desenvolvidos por Bilgren e Holmen (2008), Friedman e Mason (2004) e Hsieh (2009).

Verifica-se que o crescente interesse sobre a utilização da temática análise de stakeholders, a partir de 2005 a periodicidade de publicação destes artigos foram intensificadas, demonstrando o potencial da teoria. Tal evolução pode ser observada no trabalho de Vieira et al. (2010). Tem-se ainda que aspectos mais específicos careçam de melhor investigação empírica sobre as motivações dos stakeholders e sua rede de relações, já que são abordadas de maneira insipiente na literatura internacional. Ao se considerar as pesquisas realizadas no contexto do turismo que são publicadas em periódicos internacionais tem-se que a mesma necessita evoluir sensivelmente considerando que nesta revisão encontraram-se apenas cinco publicações, ou seja, há um potencial a ser explorado em termos de aplicação empírica da teoria no contexto do turismo, tanto internacional quanto nacionalmente, sendo que inicialmente sugere-se iniciar as pesquisas a partir do foco central da análise de stakeholders, listados na Figura 1 desta pesquisa.

\section{REFERÊNCIAS}

Beierle, T. C. (2002). The quality of stakeholder-based decisions. Risk Analysis, 22(4), 739-749.

Billgren, C., \& Holmen, H. (2008). Approaching reality: Comparing stakeholder analysis and cultural theory in the context of natural resource management. Land Use Policy, 25(4), 550-562.

Boaventura, J. M. G., Cardoso, F. R., Silva, E. S., \& Silva, R. S. (2009). Stakeholders Theory and Theory of the Firm: a study on the hierarchy of objective-functions in Brazilian companies. Rbgn-Revista Brasileira de Gestão de Negácios, 11, 289-307.

Brown, K., Adger W. N., \& Tompkins, E. (2001). Trade-off analysis for marine protected area management. Ecological Economics, 37(3), 417-434.

Cameron, B. G., Crawley, E. F., \& Loureiro, G. (2008). Value flow mapping: Using networks to inform 
stakeholder analysis. Acta Astronautica, 62(4-5), 324-333.

Chase, L. C., Siemer, W. F., \& Decker, D. J. (2002). Designing stakeholder involvement strategies to resolve wildlife management controversies. Wildlife Society Bulletin, 30(3), 937-950.

Cheng, P., Millar, C. C., \& Choi, C. J. (2006). Organizational change in stakeholder business systems: the role of institutions. Journal of Organizational Change Management, 19(3), 383-392.

Chung, W. Y., Chen, H. C., \& Reid, E. (2009). Business Stakeholder Analyzer: An Experiment of Classifying Stakeholders on the Web. Journal of the American Society for Information Science and Technology, 60(1), 59-74.

Co, H. C., \& Barro, F. (2009). Stakeholder theory and dynamics in supply chain collaboration. International Journal of Operations \& Production Management, 29(6), 591-611.

Currie, R. R., Seaton, S., \& Wesley, F. (2009). Determining Stakeholders for Feasibility Analysis. Annals of Tourism Research [S.I.], 36(1), 41-63.

De Lopez, T. T. (2001). Deforestation in Cambodia: a stakeholder management approach. International Journal of Sustainable Development and World Ecology, 8(4), 380-394.

Fassin, Y. (2008). Imperfections and shortcomings of the stakeholder model's graphical representation. Journal of Business Ethics, 80(4), 879-888.

Fassin, Y. (2009). The Stakeholder Model Refined. Journal of Business Ethics, 84(1), 113-135.

Ferrary, M. (2009). A Stakeholder's Perspective on Human Resource Management. Journal of Business Ethics, 87(1), 31-43.

Freeman, R. E. (1984). Strategic Management: A Stakeholder Approach. Massachusetts, Sage.

Friedman, A. L., \& Miles, S. (2002). Developing stakeholder theory. Journal of Management Studies, 39(1), 1-21.

Friedman, A. L., \& Miles, S. (2006). Stakeholder: theory and Practice. New York, NY: Oxford Univesity Press.

Friedman, M. T., \& Mason, D. S. (2004). A stakeholder approach to understanding economic development decision making: Public subsidies for professional sport facilities. Economic Development Quarterly, 18(3), 236-254.

Hendry, J. R. (2005). Stakeholder influence strategies: An empirical exploration. Journal of Business Ethics, 61(1), 79-99.

Hermans, L. M. (2008). Exploring the Promise of Actor Analysis for Environmental Policy Analysis: Lessons from Four Cases in Water Resources Management. Ecology and Society, 13(1).

Hermans, L. M., \& Thissen, W. A. H. (2009). Actor analysis methods and their use for public policy analysts. European Journal of Operational Research, 196(2), 808-818.

Hsieh, M. H. (2009). Human centric knowledge seeking strategies: a stakeholder perspective. Journal of Knowledge Management, 13(4), 115-133.

Jepsen, A. L., \& Eskerod, P. (2009). Stakeholder analysis in projects: Challenges in using current guidelines in the real world. International Journal of Project Management, 27(4), 335-343.

Kooskora, M. (2008). Corporate governance from the stakeholder perspective, in the context of Estonian business organizations. Baltic Journal of Management, 3(2), 193-217.

Lamberg, J. A., Pajunen, K., \& Parvinen, P. (2008). Stakeholder management and path dependence in organizational transitions. Management Decision, 46(5-6), 846-863. 
Lim, G., Ahn, H., \& Lee, H. (2005). Formulating strategies for stakeholder management: a case-based reasoning approach. Expert Systems With Applications, 28(4), 831-840.

Linkov, I., Satterstrom, F. K., \& Kiker, G. (2006). Multicriteria decision analysis: A comprehensive decision approach for management of contaminated sediments. Risk Analysis, 26(1), 61-78.

Magness V. (2008). Who are the Stakeholders Now? An Empirical Examination of the Mitchell, Agle, and Wood Theory of Stakeholder Salience. Journal of Business Ethics, 83(2), 177-192.

Medilanski, E., Chuan, L., \& Mosler, H. J. (2007). Identifying the institutional decision process to introduce decentralized sanitation in the city of Kunming (China). Environmental Management, 39(5), 648-662.

Michalski, G. V., \& Cousins, J. B. (2001). Multiple perspectives on training evaluation: Probing stakeholder perceptions in a global network development firm. American Journal of Evaluation, 22(1), 37-53.

Mitchell, R. K., Agle, B. R., \& Wood, D. J. (1997). Toward a theory of stakeholder identification and salience: Defining the principle of who and what really counts. Academy of Management Review [S.I.], 22(4), 853886.

Mitroff, I. I. (1983). Stakeholders of the organizational mind: toward a new view of organizations policy making. San Francisco, California: Jossey-Bass Publishers.

Pan, G. S. C. (2005). Information systems project abandonment: a stakeholder analysis. International Journal of Information Management, 25(2), 173-184.

Pomero, Y. R., \& Douvere, F. (2008). The engagement of stakeholders in the marine spatial planning process. Marine Policy, 32(5), 816-822.

Prell, C., Hubacek, K., \& Reed, M. (2009). Stakeholder Analysis and Social Network Analysis in Natural Resource Management. Society \& Natural Resources, 22(6), 501-518.

Reed, M. S. (2008). Stakeholder participation for environmental management: A literature review. Biological Conservation, 141(10), 2417-2431.

Reed, M. S., Graves, A., \& Dandy, N. (2009). Who's in and why? A typology of stakeholder analysis methods for natural resource management. Journal of Environmental Management, 90(5), 1933-1949.

Robson, J., \& Robson, I. (1996). From shareholders to stakeholders: Critical issues for tourism marketers. Tourism Management [S.I.], 17(7), 533-540.

Roloff, J. (2008). Learning from multi-stakeholder networks: focused stakeholder management. Journal of Business Ethics, 82(1), 233-250.

Schwarzkopf, D. L. (2006). Stakeholder perspectives and business risk perception. Journal of Business Ethics, 64(4), 327-342.

Simmons, J., \& Lovegrove, I. (2005). Bridging the conceptual divide: lessons from stakeholder analysis. Journal of Organizational Change Management, 18(5), 495-513.

Tompkins, E. L., Few, R., \& Brown, K. (2008). Scenario-based stakeholder engagement: Incorporating stakeholders preferences into coastal planning for climate change. Journal of Environmental Management, 88(4), 1580-1592.

Vandekerckhove, W., \& Dentchev, N. A. (2005). A network perspective on stakeholder management: Facilitating entrepreneurs in the discovery of opportunities. Journal of Business Ethics, 60(3), 221-232.

Varvasovszky, Z., \& Brugha, R. (2000). Stakeholder analysis: a review. Health Policy and Planning [S.I.], 15(3), 239-246.

Vidgen R. (1997). Stakeholders, soft systems and technology: Separation and mediation in the analysis of 
information system requirements. Information Systems Journal, 7(1), 21-46.

Vieira, S. F. A., Costa, B. K., Arbex, M. A., \& Carmona, V. C. (2010). Pesquisa no Turismo Utilizando Teoria dos Stakeholders: Revisando a Literatura. Seminário da Associação Nacional Pesquisa e Pós-Graduação em Turismo, São Paulo, SP, Brasil, 7.

Webb, T. J., \& Raffaelli, D. (2008). Conversations in conservation: revealing and dealing with language differences in environmental conflicts. Journal of Applied Ecology, 45(4), 1198-1204.

Williams, W., \& Lewis, D. (2008). Strategic management tools and public sector management - The challenge of context specificity. Public Management Review, 10(5), 653-671.

Wolfe, R. A., \& Putler, D. S. (2002). How tight are the ties that bind stakeholder groups? Organization Science, 13(1), 64-80.

Wong, B. (2005). Understanding stakeholder values as a means of dealing with stakeholder conflicts. Software Quality Journal, 13(4), 429-445.

Wu, X. (2007). Stakeholder identifying and positioning (SIP) models: From Google's operation in China to a general case-analysis framework. Public Relations Review, 33(4), 415-425.

Yang, J., Shen, G. Q., \& Ho, M. F. (2009). Exploring Critical Success Factors For Stakeholder Management In Construction Projects. Journal of Civil Engineering and Management, 15(4), 337-348.

Yau, O. H. M., Chow, R. P. M., \& Sin, L. Y. M. (2007). Developing a scale for stakeholder orientation. European Journal of Marketing, 41(11-12), 1306-1327.

Zakhem, A. (2008). Stakeholder management capability: A discourse-theoretical approach. Journal of Business Ethics, 79(4), 395-405. 